Diabetologe 2020 · 16:601-602

https://doi.org/10.1007/s11428-020-00655-2

Online publiziert: 29. Juli 2020

(c) Springer Medizin Verlag GmbH, ein Teil von Springer Nature 2020

\section{Originalpublikation}

Cariou B, Hadjadj S, Wargny M et al (2020) Phenotypic characteristics and prognosis of inpatients with COVID-19 and diabetes: the CORONADO study. Diabetologia 1-16 https:// doi.org/10.1007/s00125-020-05180-x

Hintergrund. Bisher liegen nur wenige Daten vor, welche Auswirkungen die Diagnose Diabetes auf den Verlauf der Coronavirus-Erkrankung-2019 („coronavirus disease 2019“, COVID-19) hat. Eine kürzlich veröffentlichte Metaanalyse von Daten aus China zeigte, dass die Diagnose Diabetes mit einem etwa 2 -fach erhöhten Risiko für eine intensivmedizinische Behandlung und einem verdreifachten Risiko für Versterben assoziiert ist [1]. In einer retrospektiven Betrachtung aus Italien konnte die Prävalenz von Diabetes mellitus bei intensivmedizinisch betreuten Patienten mit schweren Verläufen einer Infektion mit dem „severe acute respiratory syndrome corona virus 2 “(SARS-CoV-2) mit $17 \%$ angegeben werden [2], ohne dass weitere diabetesspezifische Analysen durchgeführt wurden.

In der Beobachtungsstudie CORONADO (Coronavirus SARS-CoV-2 and Diabetes Outcomes) wurden nun klinische Parameter identifiziert, die innerhalb der Gruppe der Diabetespatienten mit dem Schweregrad des Krankheitsverlaufs und dem Mortalitätsrisiko stationär behandelter COVID-19-Patienten korrelieren.

Methoden. In 53 französischen Kliniken wurden zwischen dem 10. und dem

\title{
Amrei Henn
}

Klinik und Poliklinik für Endokrinologie, Nephrologie, Rheumatologie, Universitätsklinikum Leipzig, Leipzig, Deutschland

\section{Prognosefaktoren für den Krankheitsverlauf von COVID-19 bei Diabetespatienten}

31. März 2020 Daten von insgesamt 1300 Diabetespatienten mit gesicherter COVID-19 erhoben.

Der primäre „composite endpoint“ bestand aus Intubation und Beatmung oder Tod innerhalb von 7 Tagen nach Aufnahme. Sekundäre Endpunkte waren intensivmedizinische Behandlung sowie am Tag 7 nach Aufnahme Entlassung, Intubation oder Tod.

Die erhobenen Daten beinhalteten neben klinischen Daten, wie Alter, Geschlecht und Body-mass-Index (BMI), die Klassifikation des Diabetes in die verschiedenen Typen, den aktuellsten verfügbaren Hämoglobin- $\mathrm{A}_{1 \mathrm{c}}$-Wert $\left(\mathrm{HbA}_{1 \mathrm{c}}\right)$, mikro- und makrovaskuläre Komplikationen und weitere Komorbiditäten. Zudem wurden COVID-19spezifische klinische und laborchemische Befunde (z. B. Inflammationsparameter) erhoben.

Es wurden uni- und multivariate Analysen durchgeführt, unter Einbeziehung der Diabeteskomorbiditäten einerseits und der klinischen Parameter der SARSCoV-2-Infektion andererseits.

Ergebnisse. Etwa $89 \%$ der eingeschlossenen Patienten waren Typ-2-Diabetespatienten, 3\% Typ-1-Diabetespatienten, $5 \%$ Patienten mit anderen Diabetesätiologien, bei den übrigen Patienten konnte zum Auswertungszeitpunkt keine eindeutige Zuordnung getroffen werden. In der univariaten Analyse waren ein BMI $>25 \mathrm{~kg} / \mathrm{m}^{2}$ und das männliche Geschlecht mit einem erhöhten Risiko assoziiert, den primären „composite endpoint“ zu erreichen. In der multivariaten Analyse war ein $\mathrm{BMI}>25 \mathrm{~kg} / \mathrm{m}^{2}$ der einzige Prädiktor für das Erreichen des „composite endpoint“. Alter, mikro- und makrovaskuläre Komplikationen sowie ein Schlafapnoesyndrom waren unabhängig mit dem sekundären Endpunkt Tod an Tag 7 korreliert. Der $\mathrm{HbA}_{1 \mathrm{c}}$-Wert war weder mit dem primären noch mit dem sekundären Endpunkt Tod an Tag 7 assoziiert. In Korrelation der Symptome und klinischen Parameter der COVID-19 waren Dyspnoe, Erhöhung des C-reaktiven Proteins, Lymphopenie und Niereninsuffizienz mit Erreichen des primären Endpunkts assoziiert.

\section{Kommentar}

Die aktuellen Daten geben einen wichtigen Überblick über Prognosefaktoren bei stationär behandelten Diabetespatienten mit COVID-19. Bisher lagen ausschließlich Daten aus China vor, wo sich Diabetesprävalenzen und Komorbiditäten von denen in Europa unterscheiden [3].

In der hier vorgestellten Studie stellte sich der BMI, interessanterweise aber nicht der $\mathrm{HbA}_{1 \mathrm{c}}$, als prognoserelevant heraus. So scheint nicht die generelle Blutzuckereinstellung, sondern vielmehr die aus dem Diabetes resultierenden Komorbiditäten für den Krankheitsverlauf eine entscheidende Rolle $\mathrm{zu}$ spielen. Übergewichtige Patienten haben ein höheres Risiko für die Entwicklung von Komorbiditäten, was hier eine Rolle spielen kann. Die Datenerhebung erfolgte retrospektiv und ausschließlich bei stationären Patienten. Nur bei etwa zwei Dritteln der Patienten lag ein aktueller $\mathrm{HbA}_{1 \mathrm{c}}$-Wert vor, sodass ein prospek- 
tives Studiendesign, ausgelegt auf die
Analyse diabetesspezifischer Parameter mit entsprechend fokussierter Datenerhebung zur weiteren Spezifizierung prognoserelevanter Faktoren hilfreich wäre. Zudem wäre ein Vergleich mit ambulant behandelten Diabetespatienten interessant. Des Weiteren wurden die Patienten nur bis zum 7. stationären Tag verfolgt, im Langzeitverlauf hätten ggf. weitere prognoserelevante Faktoren identifiziert werden können.

Es ist nicht auszuschließen, dass die erhebliche Belastung des Gesundheitssystems in Frankreich, die zu dem Zeitpunkt der Datenerhebung regional bestand, einen Einfluss auf die Ergebnisse hatte.

Zuletzt wäre ein Vergleich von Patienten mit und ohne Diabetes interessant, um zukünftig differenzieren zu können, ob bereits das Vorliegen eines Diabetes allein ein Risiko für einen schweren Verlauf von COVID-19 ist.

\section{Fazit für die Praxis}

- Ein erhöhter BMI ab $>\mathbf{2 5} \mathrm{kg} / \mathrm{m}^{2}$ stellte sich als unabhängiger Prognosefaktor für einen schweren Krankheitsverlauf heraus.

- Bei älteren Diabetespatienten und bei Patienten mit bestehenden diabetesassoziierten Komplikationen scheint ein erhöhtes Mortalitätsrisiko vorzuliegen.

\section{Korrespondenzadresse}

\section{Dr. med. Amrei Henn}

Klinik und Poliklinik für Endokrinologie, Nephrologie, Rheumatologie, Universitätsklinikum Leipzig

Liebigstr. 20, 04103 Leipzig, Deutschland amrei.henn@medizin.uni-leipzig.de

Interessenkonflikt. A. Henn gibt an, dass kein Interessenkonflikt besteht.

\section{Literatur}

1. Roncon L, Zuin M, Rigatelli G, Zuliani G (2020) Diabetic patients with COVID-19 infection are at higher risk of ICU admission and poor short-term outcome. J Clin Virol 127:104354. https://doi.org/ 10.1016/j.jcv.2020.104354

2. Grasselli G, Zangrillo A, Zanella A et al (2020) Baseline characteristics and outcomes of 1591 patients infected with SARS-coV-2 admitted to ICus of the Lombardy region, Italy. JAMA 323(16):1574-1581. https://doi.org/10.1001/ jama.2020.5394

3. https://www.diabetesatlas.org/upload/resources/ material/20200302_133351_IDFATLAS9e-finalweb.pdf. Zugegriffen:24. Juni 2020
Aktuelle Informationen

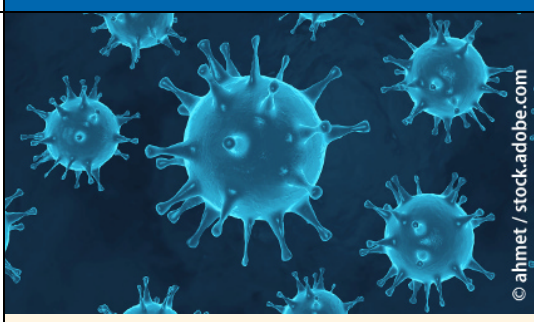

\section{Aktuelle Entwicklung zu COVID-19 bei Springer Nature und Springer Medizin}

Springer Nature und Springer Medizin unterstützen die globale Reaktion auf die COVID-19-Pandemie, indem ein schneller und direkter $\mathrm{Zu}$ gang zu den neuesten verfügbaren Forschungsergebnissen und Daten ermöglicht wird.

Auf der Homepage SpringerMedizin.de finden Sie ein immer aktuelles Dossier mit Beiträgen, Forschungsarbeiten und Ergebnissen zu SARS-CoV-2 sowie relevanten Links.

Darin z.B. auch die kürzlich publizierte

Empfehlung von DIVI, DGIIN, DGAI und DGP zur Intensivtherapie von Patienten mit COVID-19.

Springer Nature arbeitet mit globalen Organisationen zusammen, und verlinkt über SpringerNature.com/de auf eine eigene Landingpage mit einer Vielzahl an Information sowie freiem Zugriff auf die COVID-19-Contentplattformen von Nature Research, BioMed Central (BMC) und Springer.

Das Dossier zu Coronavirus / Covid-19 von Springer Medizin finden Sie hier: www.springermedizin.de/covid-19

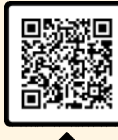

